\title{
Laparoscopic Adrenalectomy Is Beneficial for the Health-Related Quality of Life of Older Patients with Primary Aldosteronism
}

\author{
Yoshihide Kawasaki $^{a}$ Shigeto Ishidoya ${ }^{b}$ Ryo Morimoto ${ }^{c}$ Yoshikiyo Ono $^{c}$ \\ Kei Omatac Yuta Tezukac Naoki Kawamorita ${ }^{\mathrm{c}}$ Shinichi Yamashita ${ }^{a}$ \\ Koji Mitsuzuka ${ }^{a}$ Fumitoshi Satoh $^{d}$ Akihiro Ito $^{a}$ \\ aDepartment of Urology, Tohoku University Graduate School of Medicine, Sendai, Japan; b Department of Urology, \\ Sendai City Hospital, Sendai, Japan; 'Department of Nephrology, Endocrinology and Vascular Medicine, Tohoku \\ University Graduate School of Medicine, Sendai, Japan; 'Department of Clinical Endocrinology and Metabolism, \\ Tohoku University Graduate School of Medicine, Sendai, Japan
}

\section{Keywords \\ Laparoscopic adrenalectomy - Health-related quality of life . Primary aldosteronism - Medical Outcomes Study 36-Item Short-Form Health Survey · National standard value}

\begin{abstract}
Objective: Laparoscopic adrenalectomy (LADX) improves hypertension in patients with primary aldosteronism (PA). However, the antihypertensive impact of LADX appears restricted in older patients with PA. In this study, we evaluated the impact of LADX in older patients focusing on the healthrelated quality of life (HRQoL). Methods: A total of 156 patients with PA who underwent LADX in a single institution were enrolled in this prospective cohort study. The patients were divided into 2 groups, with a boundary of 60 years. The HRQoL was evaluated using the Medical Outcomes Study's 36-Item Short-Form Health Survey version 2 (SF-36v2) questionnaire before and after LADX. Demographics, clinical features, antihypertensive drugs before and after surgery, and perioperative evaluation were recorded. We compared all scale scores and summed scores between groups. Multivariate regression models were used to determine the associations between various covariables and the HRQoL. Results:
\end{abstract}

In the older PA patients, most subscales of HRQoL at baseline were lower than the national standard values. The antihypertensive drug-free rate by LADX was only $21 \%$ in older patients, compared to $58 \%$ in younger patients. However, a significant improvement in mental HRQoL was observed after LADX ( $p=0.002)$. The much preoperative antihypertensive drugs, lower preoperative potassium level, and smaller degree of comorbidities were predictors of improved mental HRQoL by LADX on multivariate analyses. Conclusion: The older PA patients showed lower mental HRQOL than the national standard populations. Although antihypertensive effects were limited for these patients, LADX was beneficial as PA treatment via improvement of mental HRQoL.

(c) 2021 The Author(s)
Published by S. Karger AG, Basel

\section{Introduction}

To date, the clinical and biochemical responses to laparoscopic adrenalectomy (LADX) have been welldescribed, and unilateral LADX results in the cure or improvement of hypertension and the correction of hypokalemia $(K \leq 3.5 \mathrm{mEq} / \mathrm{L})$ in patients with unilateral primary aldosteronism (PA) [1]. The current guidelines karger@karger.com www.karger.com/uin

Karger $\stackrel{\text { ' }}{5}$

BOPEN ACCESS
(C) 2021 The Author(s)

Published by S. Karger AG, Basel

This is an Open Access article licensed under the Creative Commons Attribution-NonCommercial-4.0 International License (CC BY-NC) (http://www.karger.com/Services/OpenAccessLicense), applicable to the online version of the article only. Usage and distribution for commercial purposes requires written permission.
Correspondence to:

Yoshihide Kawasaki, kawasaki@uro.med.tohoku.ac.jp 
Fig. 1. Patient selection in this study. PA, primary aldosteronism; HRQoL, healthrelated quality of life; LADX, laparoscopic adrenalectomy.

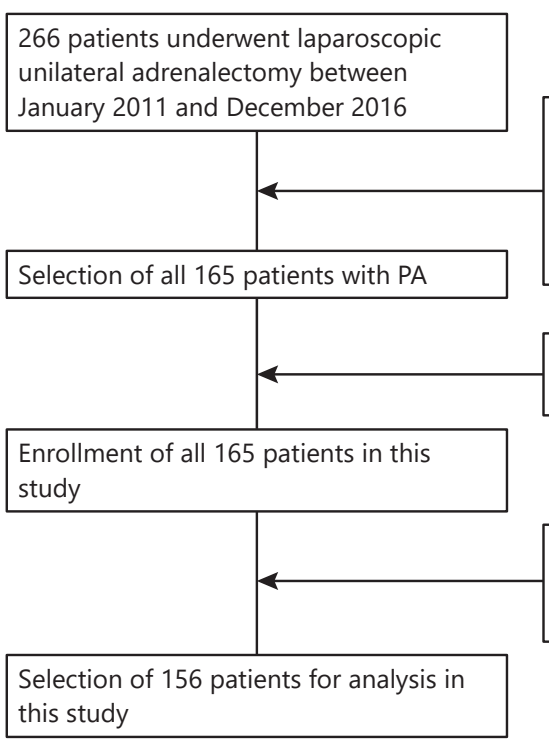

Primary aldosteronism ( $n=165)$ Subclinical cushing/Cushing syndrome $(n=60)$

Pheochromocytoma $(n=24)$

Others $(n=27)$

Enrolled if:

Patients consented to inclusion in this study

Selected if:

Patients answered questionnaires on HRQOL both before and after LADX recommend active screening for and diagnosis of $\mathrm{PA}$ in those with hypertension, and LADX is recommended for PA patients with unilateral lesions as standard care [2].

According to our clinical experience, the impact of LADX on the cure or improvement of hypertension appears somewhat restricted in older patients with PA [3]. With regard to the effects of LADX on the cure of hypertension, further evaluation of the influence of advanced age on patients with PA is needed to clarify whether to perform LADX.

Meanwhile, PA is reportedly associated with a higher prevalence of anxiety and depression $[4,5]$. Some cardiovascular factors might also play a role in depression and anxiety [6]. Previously, we reported the favorable impacts of LADX on the health-related quality of life (HRQoL) using the medical Outcomes Study's Short-Form 36-Item Health Survey version 2 (SF-36v2) questionnaire [7-9]. HRQoL is generally lower in older individuals because of the greater frequencies of diabetes mellitus (DM) and cardiovascular disease (CVD), as well as the use of a greater number of drugs. Thus, the subjective improvement of HRQoL needs to be investigated to consider other comprehensive treatments for older patients with PA. We hypothesized that PA itself would likely lower the HRQoL, particularly in older patients. To advance our previous study, this prospective study evaluated the influence of PA on HRQoL in older patients as well as the impact of LADX on HRQoL.

\section{Materials and Methods}

After institutional review board approval (approval no. 09156), informed consent was obtained from all patients with PA, and we reviewed prospectively collected data on patients. Of the 165 patients who underwent unilateral LADX for unilateral PA at Tohoku University Hospital, Sendai, from January 2011 to December 2016, the individuals ( $n=156,95 \%$ ) who responded to the questionnaire before and 6 months after LADX were enrolled in this study (Fig. 1). The criteria for PA and its subtype differentiation used in this study were based on the Japanese PA guidelines, as previously described [10]. In brief, all patients were initially screened using the plasma aldosterone/renin ratio, and unilateral PA was subsequently confirmed by fludrocortisone suppression testing and adrenal venous sampling $[3,10]$. The demographic data included the duration of hypertension and preoperative comorbidities, and the clinical data examined included the pre- and postoperative blood pressure, plasma potassium levels, and use of antihypertensive drugs. Our LADX procedures have been previously described in detail $[11,12]$. We chose an arbitrary cutoff of 60 years by quintiles for comparison. We compared all scale scores and summed scores of HRQoL between the young patient group ( $<60$ years old, $n=100$ ) and the older patient group ( $\geq 60$ years old, $n=56)$.

\section{SF-36v2 Questionnaire and Data Collection}

The demographic data, pre- and postoperative outcomes, and HRQoL were examined. HRQoL was assessed using the SF-36v2 questionnaire at baseline and at $6,12,18$, and 24 months after LADX $[8,9]$. The SF-36v2 has been validated in general populations internationally [13]. Items included in the SF-36v2 can be grouped into 8 subscales: physical functioning $(\mathrm{PF})$, social functioning (SF), role limitations due to physical problems, role physical (RP), role limitations due to emotional problems, role emotional (RE), vitality (VT), bodily pain (BP), general health $(\mathrm{GH})$ 
Table 1. Preoperative clinical characteristics and antihypertensive effect after LADX

\begin{tabular}{|c|c|c|c|c|}
\hline Median age, years (IQR) & $55(46,60)$ & $49(40,53)$ & $64(61,67)$ & $<0.001$ \\
\hline Male, $n(\%)$ & $76(49)$ & $50(50)$ & $34 / 22(60.7)$ & - \\
\hline Median duration of hypertension, years (IQR) & $9(0-40)$ & $5(3,12)$ & $18(9.5,-24.5)$ & $<0.001$ \\
\hline $\mathrm{DM}, n(\%)$ & $25(16)$ & $20(20)$ & $12(21.4)$ & 0.52 \\
\hline CVD, $n(\%)$ & $8(5)$ & $6(6)$ & $10(17.9)$ & 0.025 \\
\hline Pre-op hypokalemia, $n(\%)$ & $54(34.6)$ & $36(36)$ & $16(28.6)$ & 0.68 \\
\hline Median pre-op antihypersensitive, drugs/day (range) & $6.5(0-16)$ & $6.62(0-14)$ & $8.26(1-16)$ & 0.042 \\
\hline Median post-op antihypersensitive, drugs/day (range) & $1(0-9)$ & $1.16(0-7)$ & $1.86(0-9)$ & 0.078 \\
\hline Patients free from post-op antihypersensitive drugs, $n(\%)$ & $62(40)$ & $58(58)$ & $21(37.5)$ & $<0.001$ \\
\hline
\end{tabular}

LADX, laparoscopic adrenalectomy; DM, diabetes mellitus; CVD, cardiovascular disease.

perceptions, and general mental health (MH). These 8 items can be summarized into 2 broad categories: physical summary score (PCS), constructed using the results for PF, RP, BP, GH, and VT; and mental summary score (MCS), constructed using GH, VT, SF, $\mathrm{RE}$, and $\mathrm{MH}$. Each score ranges from 0 (poorest health status) to 100 (best health status). This study calculated scores normalized to Japanese national standard values (norm-based scores) for comparison with subscales and summary scores from the general Japanese population. In norm-based scoring, 50 points is a national standard value for Japanese individuals at each age. All subscales and summary scores were compared with published normative values for the Japanese population [13]. Norm-based SF-36v2 scores for the general Japanese population were calculated from a 2007 nationwide investigation based on Japanese population statistics from 2005.

\section{Statistical Analysis}

Data were analyzed and compared using JMP for Windows version 14.0 (SPSS Inc., Chicago, IL, USA). Data are expressed as mean \pm SE, unless otherwise specified. Differences between preand postoperative antihypertensive drugs, biochemical parameters, and SF-36v2 scores were assessed using the Mann-Whitney U test. Comparison of SF-36v2 scores between age-groups and published norm-based scores (normative values) for Japan were performed using $t$-tests. Statistical significance was accepted when the 2 -tailed $p$ value was $<0.05$. On multivariate analysis, logistic regression modeling was adopted.

\section{Results}

Preoperative Clinical Characteristics of Study Patients and Antihypertensive Effects of LADX

The preoperative clinical characteristics and antihypertensive effects of LADX are shown in Table 1. The median age was 55 (IQR 46-60) years, and the median duration of hypertension was 9 (IQR 3-17.25) years.
The median number of pre- and postoperative antihypertensive drugs was 6.5 (IQR 6-9) and 1 (IQR 1-2) years, respectively. When compared between the 2 agegroups, the median duration of hypertension was shorter in the young group than in the older group (5.5 vs. 17.5 years, respectively), and the antihypertensive drugfree rate (complete cure rate of hypertension) was higher in the young patient group than in the older patient group ( $58 \%$ vs. $21 \%$, respectively). All 156 patients used mineralocorticoid receptor (MR) antagonists before surgery, and none of the patients used MR antagonists after LADX.

\section{Subscales of SF-36v2 Based on National Standard}

Values in the 2 Age-Groups

Subscales of SF-36v2 at baseline were evaluated in all patients by preoperative factors, including sex, DM, history of CVD, serum potassium level, and duration of hypertension. No significant differences in baseline SF-36v2 subscale scores were found for comorbidities (DM and CVD), preoperative serum potassium level, or duration of hypertension (data not shown).

Comparing subscales of SF-36v2 between the 2 agegroups, in the young patient group, all subscales except $\mathrm{BP}$ at baseline were lower than the national standard values (norm-based score). However, SF, RE, MH, and PF at 24 months after LADX were higher than the national standard values (Fig. 2a). In the older group, PF, RP, $\mathrm{GH}$, and $\mathrm{RE}$ at baseline were lower than the national standard values. Although PF, RF, and RE were still under the national standard values at 24 months after LADX, VT, SF, and MH were over the national standard values (Fig. 2b). 
Improvement in Summary Component Scores of SF-36 $v 2$ by LADX

As mentioned earlier, in terms of scoring with SF$36 \mathrm{v} 2$, the 8 subscales can be summarized into the $2 \mathrm{com}$ ponents of PCS and MCS. Preoperative clinical characteristics and antihypertensive effects from LADX in these 2 age-groups are shown in Table 1. PCS at baseline was significantly lower in older patients than in young patients $(p=0.002)$. PCS in young patients decreased gradually for 24 months after LADX, but even so, PCS at 24 months after LADX was not significantly lower than that at baseline (Fig. 3a). However, the reduction in PCS from baseline was found to be larger in older patients than in young patients. PCS in older patients decreased gradually for 24 months after LADX, and PCS at 18 and 24 months after LADX was significantly lower than that at baseline $(p=$ 0.031 and $p=0.048$, respectively). MCS at 12 months after LADX was significantly improved compared to that at baseline in both age-groups. MCS was higher in older patients than in young patients at baseline and improved gradually for 24 months after LADX in older patients (Fig. 3b).

Predictors associated with improvement of MCS at 24 months after LADX in older patients. On univariate analyses, age $(p=0.048)$, CCI $(p=0.041)$, preoperative serum potassium level $(p=0.036)$, and preoperative MCS $(p=$ 0.002 ) were significant predictors for improved MCS at 24 months after LADX in our prospective cohort study (Table 2). Sex, BMI, duration of hypertension, history of CVD, and the number of antihypertensive drugs before and after LADX were not predictors for MCS improvement.

On multivariate analyses, the number of preoperative antihypertensive drugs, preoperative potassium level, and CCI were independent predictors of MCS improvement after LADX (Table 2), while sex, BMI, duration of hypertension, and number of postoperative antihypertensive drugs were not predictors. As supplementary information, the cure rate of hypertension after LADX was not found to significantly influence MCS in either agegroups.

\section{Discussion/Conclusion}

SF-36 scores in patients with PA were found to be lower than the national standard value (norm-based score) $[8,9]$. However, few studies have focused on the HRQoL of PA patients, especially older patients treated with LADX $[1,14]$.

LADX for HRQoL of Older PA Patients

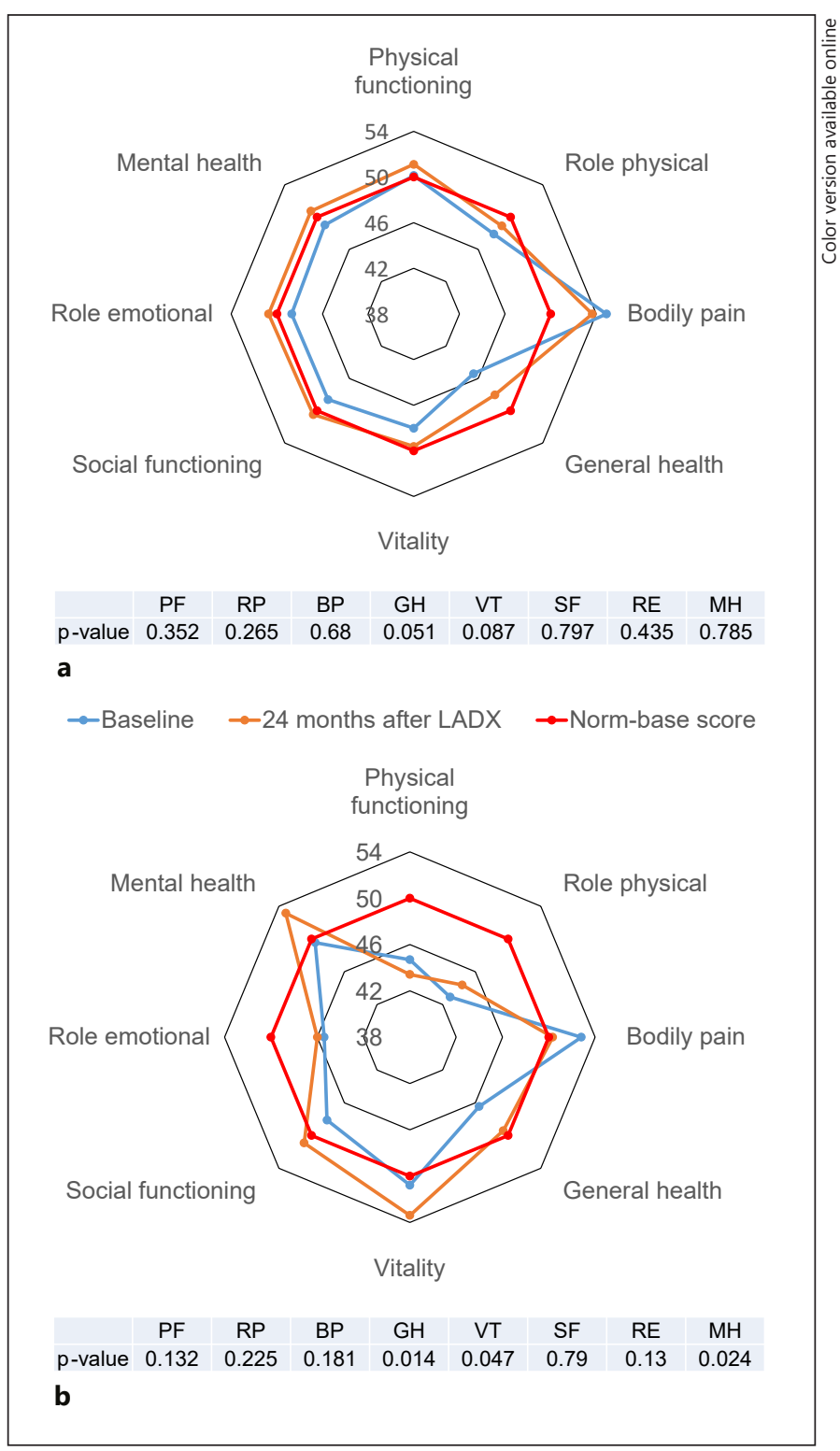

Fig. 2. Subscales of SF-36v2 in the 2 age-groups. The red line shows a norm-based score of 50 points for the general population in our country. The blue line shows subscales at baseline, and the orange line shows subscales at 24 months after LADX in young patients (a) and older patients (b). A $p$ value $\left(^{*}\right)$ is showed as statistical significance between baseline scores of the patients and norm-based score of the general population. LADX, laparoscopic adrenalectomy; SF-36v2, Short-Form 36-Item Health Survey version 2; PF, physical functioning; SF, social functioning; RP, role physical; RE, role emotional; VT, vitality; $\mathrm{BP}$, bodily pain; $\mathrm{GH}$, general health; $\mathrm{MH}$, mental health.

In this study, we found that LADX was beneficial to older patients with low preoperative MCS, who suffered from long-term comorbidities and hypertension. Deterioration of mental HRQoL can be the result of nonspe- 


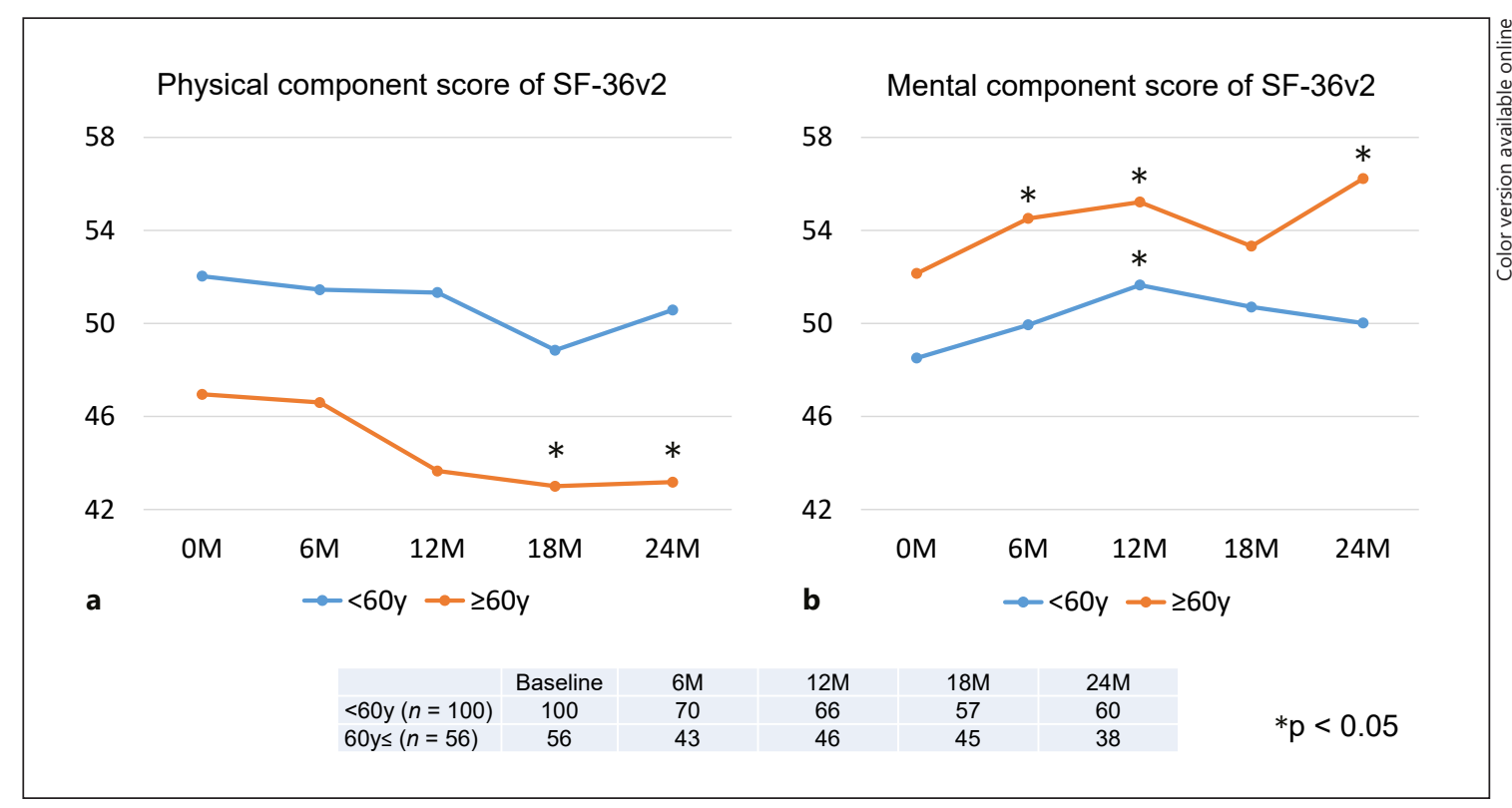

Fig. 3. Changes in summary component scores of SF-36v2 from baseline to 24 months after LADX in the 2 agegroups. a Physical summary component score. $\mathbf{b}$ Mental summary component score. A $p$ value $\left(^{*}\right)$ is showed as statistical significance of scores at each time compared to scores at 0 month $(0 \mathrm{M})$ of the patients. LADX, laparoscopic adrenalectomy; SF-36v2, Short-Form 36-Item Health Survey version 2; MCS, mental summary score; PCS, physical summary score.

Table 2. Uni- and multivariate analyses of preoperative factors on the improvement of the postoperative MCS

\begin{tabular}{|c|c|c|c|c|c|c|}
\hline & \multicolumn{3}{|c|}{ Univariate } & \multicolumn{3}{|c|}{ Multivariate } \\
\hline & OR & $95 \% \mathrm{CI}$ & $p$ value & OR & $95 \% \mathrm{CI}$ & $p$ value \\
\hline Age, years & 1.1 & $0.97-0.99$ & 0.048 & 1.97 & $0.02-1.68$ & 0.054 \\
\hline Female sex & 1.27 & $0.57-2.84$ & 0.056 & 1.17 & $0.57-1.84$ & 0.052 \\
\hline BMI & 0.98 & $0.88-1.1$ & 0.760 & 0.97 & $0.3-1.56$ & 0.846 \\
\hline Duration of hypertension, years & 1.02 & $0.98-1.1$ & 0.370 & 1.10 & $0.98-1.2$ & 0.113 \\
\hline CVD & 2.27 & $0.36-14.2$ & 0.382 & - & - & - \\
\hline CCI & 0.23 & $1.05-1.23$ & 0.041 & 0.78 & $1.45-1.95$ & $<0.001$ \\
\hline Preoperative potassium, $<3.5 \mathrm{mEq} / \mathrm{L}$ & 1.12 & $1.01-1.12$ & 0.036 & 1.96 & $1.08-1.82$ & 0.0015 \\
\hline Preoperative antihypertensive drugs, $n$ & 0.1 & $0.97-1.22$ & 0.103 & 0.37 & $1.02-1.78$ & 0.018 \\
\hline Postoperative antihypertensive drugs, $n$ & 1.03 & $0.83-1.28$ & 0.801 & 1.26 & $1.23-1.81$ & 0.154 \\
\hline Preoperative MCS & 1.92 & $1.03-1.14$ & 0.002 & - & - & - \\
\hline
\end{tabular}

MCS, mental summary score; CVD, cardiovascular disease.

cific symptoms associated with hypertension [15] as well as depression and anxiety due to excess aldosterone, especially in female patients with PA [1]. Although we concluded that normalizing the plasma aldosterone level by LADX contributed to the improvement of mental HRQoL in older patients, the placebo effect of LADX for improvement of MCS was possible.
Lumachi et al. [16] described that comorbidities and a longer duration of hypertension are major factors in preventing the complete cure of hypertension in patients with PA after LADX. So that older patients indicated a lower cure rate of hypertension, in our results regarding HRQoL, DM, CVD, and the cure rate were not important factors influencing the improvement of postoperative 
MCS. In this study, preoperative hypokalemia, which is associated with muscle weakness, cramps, paresthesia, palpitations, constipation, polyuria, and nocturia [15], fewer preoperative antihypertensive drugs and lower CCI were also found to be predictors for the improvement of postoperative MCS by LADX.

MR antagonists have been reported to improve the HRQoL in patients with PA [17]. The combination of antihypertensive drugs and MR antagonists improves not only blood pressure but also HRQoL in many patients [18]. However, the adverse effects of some antihypertensive drugs, including MR antagonists, could exert a negative influence on the mental HRQoL [19-22]. As we know, normalizing plasma aldosterone by LADX could make patients free from MR antagonists. Our results revealed that the correction of excessive plasma aldosterone by LADX reduced the anxiety of patients with possible adverse effects from MR antagonists.

PA is associated with cardiovascular hypertrophy and fibrosis, as well as renal damage independent of blood pressure levels; these changes can sometimes be irreversible, emphasizing the need for prompt treatment $[23,24]$. Generally, in most patients, hypertension is resolved 1-6 months after adrenalectomy [25]. The most important predictors of persistent hypertension include older age, comorbidities, and longer duration of hypertension before surgery [16]. So a lower CCI (fewer comorbidities) was understandable as a predictor of MCS improvement because the general condition of patients with a higher CCI and many comorbidities was likely to remain MCS unchanged before and after LADX. However, in older patients with a longer duration of hypertension, our study found that LADX itself improved mental HRQoL.

Some limitations of our study should be considered. First, as our study only included patients who were eligible for LADX in a single institution, so selection bias did exist. Second, we could not compare the HRQoL of patients who received medical treatment without LADX. Despite these limitations, LADX is amenable to PA patients with uncontrollable blood pressure and hypokalemia who received even sufficient hypertensive drugs and MR agents.

In conclusion, through our study, we found that in the older PA patients, preoperative mental HRQOL were lower than the national standard values. We emphasize that normalizing plasma aldosterone levels by LADX is encouraged to undergo for older PA patients with preexisting comorbidities as a means to improve mental HRQoL.

\section{Acknowledgements}

Study processes, including data collection and management, were performed by Natsue Abe, a scientific officer at the Department of Urology, Tohoku University Graduate School of Medicine, Sendai. We would like to thank Editage (www.editage.com) for English language editing.

\section{Statement of Ethics}

This study was approved by Tohoku University Review Board (approval no. 09-156). Informed consent was obtained from all patients with PA, and we reviewed prospectively collected data on patients.

\section{Conflict of Interest Statement}

The authors have no conflicts of interest to declare.

\section{Funding Sources}

This study was supported in part by Grants-in-Aid for Scientific Research from the Japanese Ministry of Education, Culture, Sports, Science, and Technology (Grant Nos. 16K11062 and 20K07582).

\section{Author Contributions}

Conception or design of the work: Yoshihide Kawasaki and Shigeto Ishidoya. Interpretation of data for the work: Ryo Morimoto, Yoshikiyo Ono, Kei Omata, Yuta Tezuka, Naoki Kawamorita, Shinichi Yamashita, Koji Mitsuzuka, Fumitoshi Satoh, and Akihiro Ito. Drafting the paper: Yoshihide Kawasaki, and Shigeto Ishidoya. Critical revision of the paper: Yoshihide Kawasaki and Shigeto Ishidoya.

\section{Data Availability Statement}

The data that support the findings of this study are available from the corresponding author (Yoshihide Kawasaki), upon reasonable request, considering the ethical background of the patients.

\begin{tabular}{|c|c|}
\hline References & $\begin{array}{l}1 \text { Sukor N, Kogovsek C, Gordon RD, Robson D, } \\
\text { Stowasser M. Improved quality of life, blood } \\
\text { pressure, and biochemical status following } \\
\text { laparoscopic adrenalectomy for unilateral } \\
\text { primary aldosteronism. J Clin Endocrinol } \\
\text { Metab. 2010 Mar;95(3):1360-4. } \\
2 \text { Funder JW, Carey RM, Mantero F, Murad } \\
\text { MH, Reincke M, Shibata H, et al. The man- } \\
\text { agement of primary aldosteronism: case de- } \\
\text { tection, diagnosis, and treatment: an endo- } \\
\text { crine society clinical practice guideline. J Clin } \\
\text { Endocrinol Metab. } 2016 \text { May;101(5):1889- } \\
\text { 916. }\end{array}$ \\
\hline
\end{tabular}


3 Ishidoya S, Kaiho Y, Ito A, Morimoto R, Satoh F, Ito S, et al. Single-center outcome of laparoscopic unilateral adrenalectomy for patients with primary aldosteronism: lateralizing disease using results of adrenal venous sampling. Urology. 2011 Jul;78(1):68-73.

4 Emanuele E, Geroldi D, Minoretti P, Coen E, Politi P. Increased plasma aldosterone in patients with clinical depression. Arch Med Res. 2005 Sep;36(5):544-8.

5 Sonino N, Tomba E, Genesia ML, Bertello C, Mulatero P, Veglio F, et al. Psychological assessment of primary aldosteronism: a controlled study. J Clin Endocrinol Metab. 2011 Jun;96(6):E878-83.

6 Funder JW, Reincke M. Aldosterone: a cardiovascular risk factor? Biochim Biophys Acta. 2010 Dec;1802(12):1188-92.

7 Ishidoya S, Kawasaki Y, Namiki S, Morimoto $\mathrm{R}$, Takase K, Ito A. Changes in quality of life after laparoscopic adrenalectomy for patients with primary aldosteronism: prospective 2 -year longitudinal cohort study in a Japanese tertiary center. Int J Urol. 2019 Jul;26(7):7523.

8 Fukuhara S, Bito S, Green J, Hsiao A, Kurokawa K. Translation, adaptation, and validation of the SF-36 health survey for use in Japan. J Clin Epidemiol. 1998 Nov;51(11): 1037-44.

9 Fukuhara S, Ware JE Jr, Kosinski M, Wada S, Gandek B. Psychometric and clinical tests of validity of the Japanese SF-36 health survey. J Clin Epidemiol. 1998 Nov;51(11):1045-53.

10 Satoh F, Abe T, Tanemoto M, Nakamura M, Abe M, Uruno A, et al. Localization of aldosterone-producing adrenocortical adenomas: significance of adrenal venous sampling. Hypertens Res. 2007 Nov;30(11):1083-95.

11 Higashihara E, Tanaka Y, Horie S, Aruga S, Nutahara K, Minowada S, et al. Laparoscopic adrenalectomy: the initial 3 cases. J Urol. 1993 May;149(5):973-6.
12 Jeong BC, Park YH, Han DH, Kim HH. Laparoendoscopic single-site and conventional laparoscopic adrenalectomy: a matched casecontrol study. J Endourol. 2009 Dec;23(12): 1957-60.

13 Suzukamo Y, Fukuhara S, Green J, Kosinski M, Gandek B, Ware JE. Validation testing of a 3-component model of short form-36 scores. J Clin Epidemiol. 2011 Mar;64(3): 301-8.

14 Kunzel HE, Apostolopoulou K, Pallauf A, Gerum S, Merkle K, Schulz S, et al. Quality of life in patients with primary aldosteronism: gender differences in untreated and longterm treated patients and associations with treatment and aldosterone. J Psychiatr Res. 2012 Dec;46(12):1650-4.

15 Hamer M, Batty GD, Stamatakis E, Kivimaki M. Hypertension awareness and psychological distress. Hypertension. 2010 Sep;56(3): 547-50.

16 Lumachi F, Ermani M, Basso SM, Armanini D, Iacobone M, Favia G. Long-term results of adrenalectomy in patients with aldosteroneproducing adenomas: multivariate analysis of factors affecting unresolved hypertension and review of the literature. Am Surg. 2005 Oct; 71(10):864-9.

17 Ahmed AH, Gordon RD, Sukor N, Pimenta E, Stowasser M. Quality of life in patients with bilateral primary aldosteronism before and during treatment with spironolactone and/or amiloride, including a comparison with our previously published results in those with unilateral disease treated surgically. J Clin Endocrinol Metab. 2011 Sep;96(9):2904-11.

18 Velema M, Dekkers T, Hermus A, Timmers $\mathrm{H}$, Lenders J, Groenewoud H, et al. Quality of life in primary aldosteronism: a comparative effectiveness study of adrenalectomy and medical treatment. J Clin Endocrinol Metab. 2018 Jan 1;103(1):16-24.
19 Lopez JF, Chalmers DT, Little KY, Watson SJ. A.E. Bennett research award. Regulation of serotonin1A, glucocorticoid, and mineralocorticoid receptor in rat and human hippocampus: implications for the neurobiology of depression. Biol Psychiatry. 1998 Apr 15; 43(8):547-73.

20 Holsboer F. The rationale for corticotropinreleasing hormone receptor (CRH-R) antagonists to treat depression and anxiety. J Psychiatr Res. 1999 May;33(3):181-214.

21 Hardeveld F, Spijker J, Peyrot WJ, de Graaf R, Hendriks SM, Nolen WA, et al. Glucocorticoid and mineralocorticoid receptor polymorphisms and recurrence of major depressive disorder. Psychoneuroendocrinology. 2015 May;55:154-63.

22 Vrijsen JN, Vogel S, Arias-Vásquez A, Franke B, Fernández G, Becker ES, et al. Depressed patients in remission show an interaction between variance in the mineralocorticoid receptor NR3C2 gene and childhood trauma on negative memory bias. Psychiatr Genet. 2015 Jun;25(3):99-105.

23 Rossi GP, Bernini G, Desideri G, Fabris B, Ferri C, Giacchetti G, et al. Renal damage in primary aldosteronism: results of the PAPY study. Hypertension. 2006 Aug;48(2):2328.

24 Sato S, Kawasaki Y, Ito A, Morimoto R, Shimada S, Sato T, et al. Improvement of cardiac function by laparoscopic adrenalectomy in a patient with severe heart failure attributable to primary aldosteronism. Tohoku J Exp Med. 2019 May;248(1):31-6.

25 Funder JW, Carey RM, Fardella C, GomezSanchez CE, Mantero F, Stowasser M, et al. Case detection, diagnosis, and treatment of patients with primary aldosteronism: an endocrine society clinical practice guideline. J Clin Endocrinol Metab. 2008 Sep;93(9): 3266-81. 